\title{
Time Frame Based Dynamic Data Shift Algorithm for Data Security in Mobile Cloud Computing
}

\author{
Sumangala B, Sidhar N K, Suresh P
}

\begin{abstract}
Mobile Computing is an Innovation in the Field of IT and it brought so many changes in the lives of Human Beings. Initially when mobile phone introduced it is a device useful only for Making and Receiving calls and Sending and Receiving Text Messages. As the technology changed drastically today's mobile world has become Smart World. Where Mobile phone is not only for calls and messages, it will be used for different purposes starting from Storing The Data, Accessing Different Applications like MS Word, MS Excel etc.., to take photos, and listening songs etc..., So the mobile era brought so many changes in the life of human being, initially people are happy with $4 G B$ memory devices now Smart Phones coming with $128 \mathrm{~GB}$ and $256 \mathrm{~GB}$ still people need more memory. As the usage of Mobile Phone is getting increased storing data in the given space is becoming limited. To overcome this cloud computing has taken one more step to connect the applications running on mobile to connect with cloud to store and process the data. Initially the Application will be using only the memory of mobile phones but now the applications are moving to cloud. To give an example the applications like DROP BOX, Google Drive, One Drive are running on their servers now they moved to cloud where people can access the application with ease of access. The major problems faced by users are Security and Accessing the Applications without any interruption. In this Research Paper the major focus is on how to improve the security of the Data and the minor focus is on ease of access to applications. In order to provide security for the data two level security system will be implemented. For ease access there are few techniques and frameworks need to be changed according to the problem.
\end{abstract}

Keywords: $\quad$ Cloud Security,Mobile,Framework..

\section{INTRODUCTION}

Mobile Computing is the trend now where there are no limits on the usage of mobiles. Initially when mobiles introduced they are used only for Text and SMS. Today's mobile could able to create and run the applications and also store and process the data. TO give an example whatever the computer professional could able to do on desktop or laptop more than $85 \%$ of the work on mobile itself like creation of Word Documents and executing programs like $\mathrm{C}, \mathrm{C}++$ and JAVA etc.., Editing the Photos and doing image processing activities. The trend of mobile phones changed from $256 \mathrm{~KB}$ to $4 \mathrm{~GB}$ and then to $16 \mathrm{~GB}$ to $32 \mathrm{~GB}$ and now people are using

Revised Manuscript Received on July 05, 2019

Sumangala B, Assistant. professor, dept of CSE, Sri Venkayeshwara College of Engineering Bangalore,

Sidhar N K, Assistant. professor, dept of E\&CE, Sri Venkayeshwara College of Engineering Bangalore,

Suresh P, Assistant. professor, dept of CSE, Sri Venkayeshwara College of Engineering Bangalore almost 256GB which is equal to a laptop storage space 5 to 6 years back. In the same way the display it is 1.5 Inch to 2.5 Inch now the mobiles are coming with 7 Inch. The following

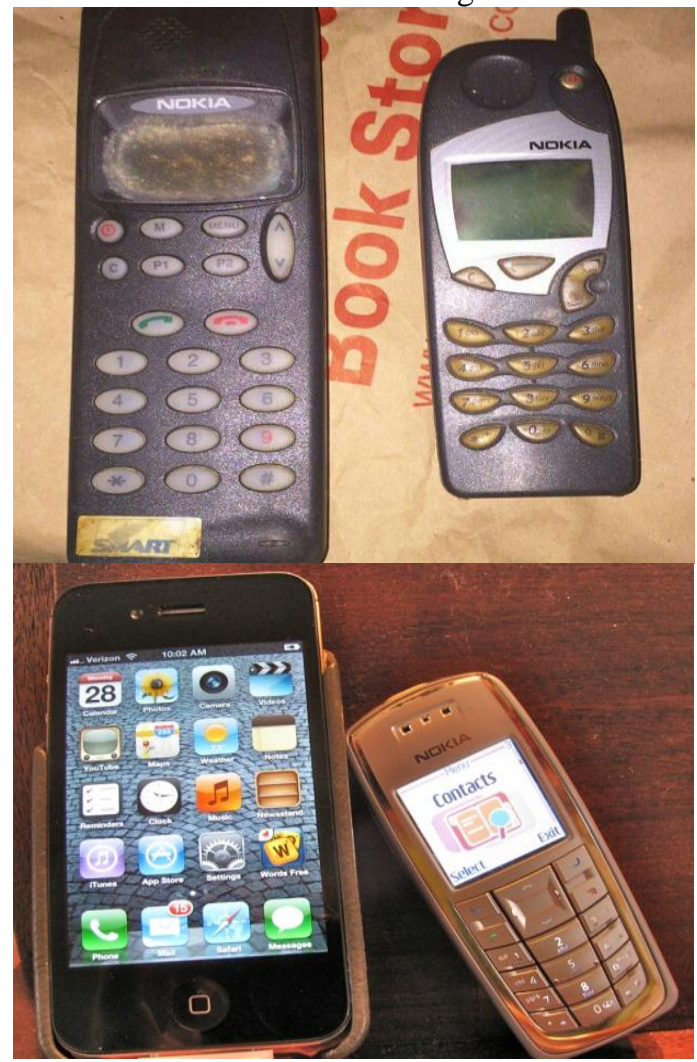

Fig (1). Old Mobile Phone Models without Android Operating System.

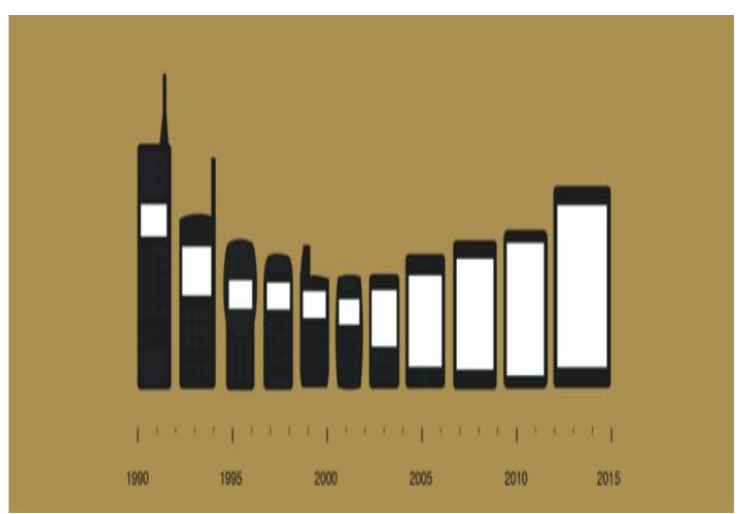

Fig (2). The Variation in Size of Mobile phones Display

Along with Screen Size and Memory the technology has changed drastically. The Mobile Computing Trend

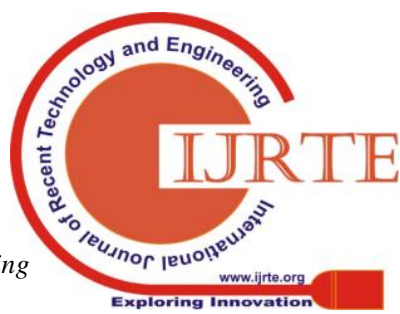


Changed with the Invention of Android Operating System. Where people started using Smart Phones and till that time people are using Mobile Phone for only Calling and Sending and Receiving Text Messages though the Size Increased with Memory.[1]

Once the Android Operating System came into the picture the entire structure of Mobile Phones changed, Started calling them as Smart Phones. Where in this Smart Phones everything will be running based on the applications.[1] The Culture of Applications Started, it increased the usage of Mobiles Phones and also opened doors for technocrats to develop applications as per the need of the customer. As Android is an opened doors not only to the Technocrats and also for the business people where so manystart-ups started to develop android applications and sell them in play store.[2]

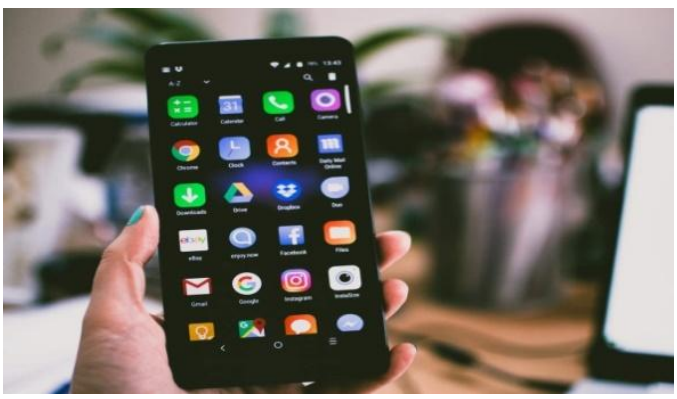

Fig (3). Android Smartphone

This is where the need of Mobile Computing has come into existence as number of apps are getting increased the need for the Storage and processor speed increased.[1]The companies started developing the different apps by creating the need among mobile users. Apart from this people are fond of Games and Social Networking sites. These are consuming so much of space which again raised the need of huge memory in the mobile.[2] The companies cannot increase memory just like that because there are some factors associated with this. The alternate for this,People started using Cloud Space in order to store the Data and process it whenver it is essential. TO give an Example all the photos and documents of the user instead of storing them on mobile phone they will be stored on cloud when and when there is a need that will be accessed and stored it back to cloud.

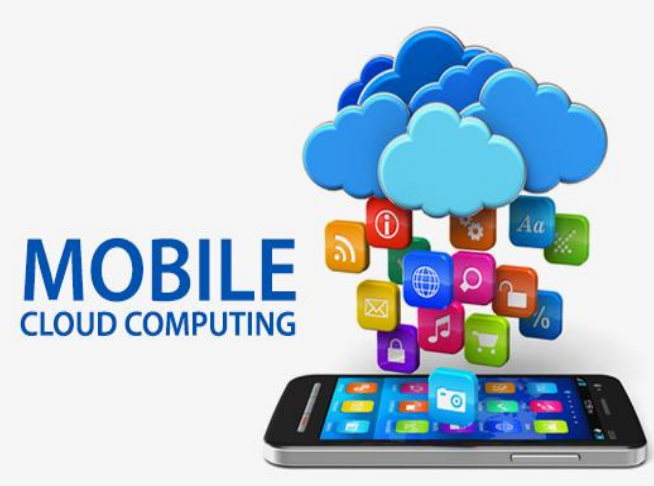

Fig (4). Mobile Cloud Computing

Now that companies also started thinking in mobile cloud computing instead of spending huge money on servers they are dependent on cloud [2] to store their applications and data. These have opened doors for the business people to save their time and money in order to perform their day to day tasks.
So along with Mobile the Cloud is also going to play a vital role for the next 15 to 20 years. The need of Mobile Cloud Computing [2] is getting increased day by day as the number of mobile users is also getting increased. [5]

Though the technology is getting increased day by day and the number of users are increased still the problems are also increasing, the main two problems faced by most of the mobile Cloud Users is Ease of Access and Security. [3]

In this paper these two issues will be addressed with an intuition solve them at most.

\section{Proposed Method for Data Security and Ease of Access in Mobile Cloud Computing:}

Data Security is one of the major issues faced by mobile cloud users. There are so many algorithms available for data security from RSA, DES etc..;[4]the main problem with these algorithms is the reverse process of encryption is decryption which means the data security is not fully achievable. The solution for this is better to have one dynamic system with time frame. As the security purely depends on the time frame here the new technique also mainly concentrates on time frame and unique key of 30 Bit Long.

The Algorithm deals with mainly time frame as well the algorithm is named as Time Frame Based Dynamic Data Shift Algorithm for Enhanced Data Security in Cloud.

\section{Time Frame Based Dynamic Data Shift Algorithm}

In this algorithm the main concept the key is fixed initially and the Dynamism will be applied when user want to access the data. How the key will be generated, the key is a combination of Data of Birth of Candidate who is using Mobile, Date of Birth of Father and Date of Birth of mother followed by Data of First Registration and Location. Just to Give an Example let consider person $\mathrm{X}$ is registering then theKey is going to be

\begin{tabular}{|l|l|l|l|l|}
\hline $\begin{array}{l}\text { DO } \\
\text { B } \\
(X)\end{array}$ & $\begin{array}{l}\text { DOB } \\
\text { (X Father) }\end{array}$ & $\begin{array}{l}\text { DOB(X } \\
\text { Mother })\end{array}$ & $\begin{array}{l}\text { Time of } \\
\text { First } \\
\text { Registrati } \\
\text { on }\end{array}$ & $\begin{array}{l}\text { Location of } \\
\text { Registration }\end{array}$ \\
\hline
\end{tabular}

This is the basic information collected from the user at the time of registration. The question arises here is it is very easy to collect Date of Birth Information of a user. Here the Dynamism is not applied at the time of collecting data it is applied at the time of processing the data.[3] And the last 6 Digits are going to be confidential always which needs to be remembered by the user i.e. registered time and location.

\section{How it Works:}

Once the user creates the Key based on his data as mentioned in the title of the Algorithm the Time Frame is going to play key role along with analytics. Suppose if a user wants to access his own data from the cloud he will be asked with few questions with time frame. If he could able to answer with in stipulated time then only he could be able to access the data. TO give an example let's consider an user want to access his personal data stored in cloud they may get questions like

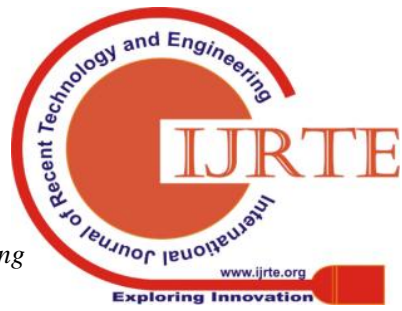


1. Type Your Date of Birth Year in Reverse order and the time frame is $30 \mathrm{Sec}$.

2. Enter the Date of Birth Data of Yours and Your Father and the time frame is $20 \mathrm{Sec}$.

3. Multiply your Date of Birth and Divide by 5 and enter the answer and the time frame is 30 Sec.

4. All 29 Characters shown in X marks and asked user to guess one Character in $5 \mathrm{sec}$.

5. The Dynamic Data will be shown in the Form multiple Bubbles and user need to identify the Correct Answer.

These are the few samples of analytics the questionnaire or set of questions will be increased based on the user experience and surveys.

This dynamism will really help mobile phone users to protect their data in the cloud and also access it whenever there is a need.

Let's consider the failure analysis if suppose someone comes to know about the Date of Birthsof three people , as discussed in the abstract the second level of verification will be there where people need to remember analytics on the 6 digits which is a combination of 4 Digits Time and 2 Digits Location. As a security measure to increase the strength the location can be increased to 4 digits as well. So, the security will be increased in each and every step this is how the algorithm works.

The entire algorithm will work as discussed above. So this will reduce the problem of security with respect to mobile cloud computing [3]. When it comes to Ease of Access of applications the network related issues need to be resolved first and the infrastructure has to be developed a way that the if internet connectivity is not there or poor the process should store data temporarily in cache once it is get into high speed network it should interact with the cloud for data process. The entire process should include both offline and online data access for cloud. The FPGA's should be attached to the mobile processors as well for performing common tasks instead of providing risk to the processor. [4]

Most of the time the data loss or theft is happening when there is slow data connectivity or when there is a drop in the pocket because of poor connectivity. This address has been issue here by processing data only when there is high speed internet connectivity and remaining time it will be accessed offline.

Though it is not a proper solution the major research in this paper is on providing data security than the network access. It may not be $100 \%$ possible to provide security. But using Time Frame based Dynamic Data Shift Algorithm the problem of security will be solved to $80 \%$.

The key size is given here is 30Bits which is not actually fixed, which is fixed as of now based on security level. The research is going on by increasing the key size and checking for multiple iterations how difficult it is to crack the key. The advantage here is if hacker hacks or cracks the key still the second level security is there like CVV of Plastic Card which will protect the data from the hackers.

\section{Conclusion:}

TO conclude the data security is an issue when people are using same kind of algorithms which are known to all the people. As per the survey the security algorithms should be developed as a system and which should differ from system to system or at least organization to organization based on the severity of security level required.

\section{Future Enhancement:}

There is need of research in the field of Ease of Accessing cloud from Mobile as well as how effectively the communication happening between Mobile and Cloud.

\section{References:}

[1]. Satyanarayana M S, Abhilash D C, Srinivas G "Data Analytics to allocate Berths in Reserved Compartments in Indian Railways" International Journal of Engineering and Technology (UAE), Vol 7 ,No. 2.33,2018,PP 764-766

[2]. R. G. Alakbarov and O. R. Alakbarov, "Mobile clouds computing: Current state, architecture and problems," 2017 Second International Conference on Electrical, Computer and Communication Technologies (ICECCT), Coimbatore, 2017, pp. 1-6.

[3]. S. Saha and M. S. Hasan, "Effective task migration to reduce execution time in mobile cloud computing," 2017 23rd International Conference on Automation and Computing (ICAC), Huddersfield, 2017, pp. 1-5

[4]. J. Zhang, Z. Zhang and H. Guo, "Towards Secure Data Distribution Systems in Mobile Cloud Computing," in IEEE Transactions on Mobile Computing, vol. 16, no. 11, pp. 3222-3235, 1 Nov. 2017.

[5].M. Bahrami, "Cloud Computing for Emerging Mobile Cloud Apps," 2015 3rd IEEE International Conference on Mobile Cloud Computing, Services, and Engineering, San Francisco, CA, 2015, pp. 4-5 